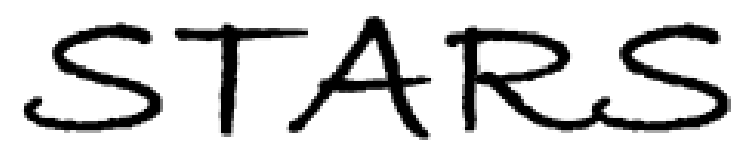

University of Central Florida

STARS

$1-1-2005$

\title{
Polar anchoring energy measurement of vertically aligned liquid- crystal cells
}

\author{
Xiangyi Nie \\ University of Central Florida \\ Yi-Hsin Lin \\ University of Central Florida \\ Thomas X. Wu \\ University of Central Florida \\ Haiying Wang \\ University of Central Florida \\ Zhibing Ge \\ University of Central Florida
}

Find similar works at: https://stars.library.ucf.edu/facultybib2000

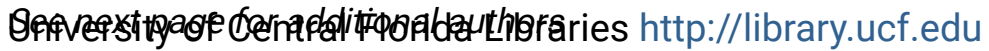

This Article is brought to you for free and open access by the Faculty Bibliography at STARS. It has been accepted for inclusion in Faculty Bibliography 2000 s by an authorized administrator of STARS. For more information, please contact STARS@ucf.edu.

\section{Recommended Citation}

Nie, Xiangyi; Lin, Yi-Hsin; Wu, Thomas X.; Wang, Haiying; Ge, Zhibing; and Wu, Shin-Tson, "Polar anchoring energy measurement of vertically aligned liquid-crystal cells" (2005). Faculty Bibliography 2000s. 5516. https://stars.library.ucf.edu/facultybib2000/5516

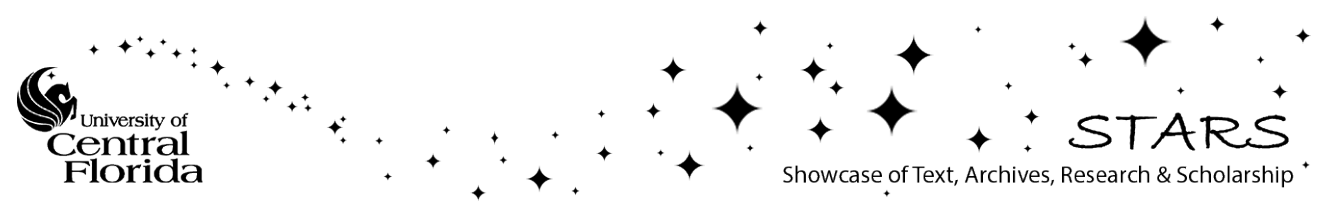




\section{Authors}

Xiangyi Nie, Yi-Hsin Lin, Thomas X. Wu, Haiying Wang, Zhibing Ge, and Shin-Tson Wu 


\section{Polar anchoring energy measurement of vertically aligned liquid-crystal cells}

Cite as: J. Appl. Phys. 98, 013516 (2005); https://doi.org/10.1063/1.1942630

Submitted: 14 February 2005. Accepted: 04 May 2005. Published Online: 07 July 2005

Xiangyi Nie, Yi-Hsin Lin, Thomas X. Wu, Haiying Wang, Zhibing Ge, and Shin-Tson Wu

\section{ARTICLES YOU MAY BE INTERESTED IN}

Anchoring energy and cell gap effects on liquid crystal response time

Journal of Applied Physics 101, 103110 (2007); https://doi.org/10.1063/1.2734870

Anchoring energy enhancement and pretilt angle control of liquid crystal alignment on polymerized surfaces

AIP Advances 5, 097218 (2015); https://doi.org/10.1063/1.4932153

Nematic polar anchoring strength measured by electric field techniques

Journal of Applied Physics 86, 4199 (1999); https://doi.org/10.1063/1.371347

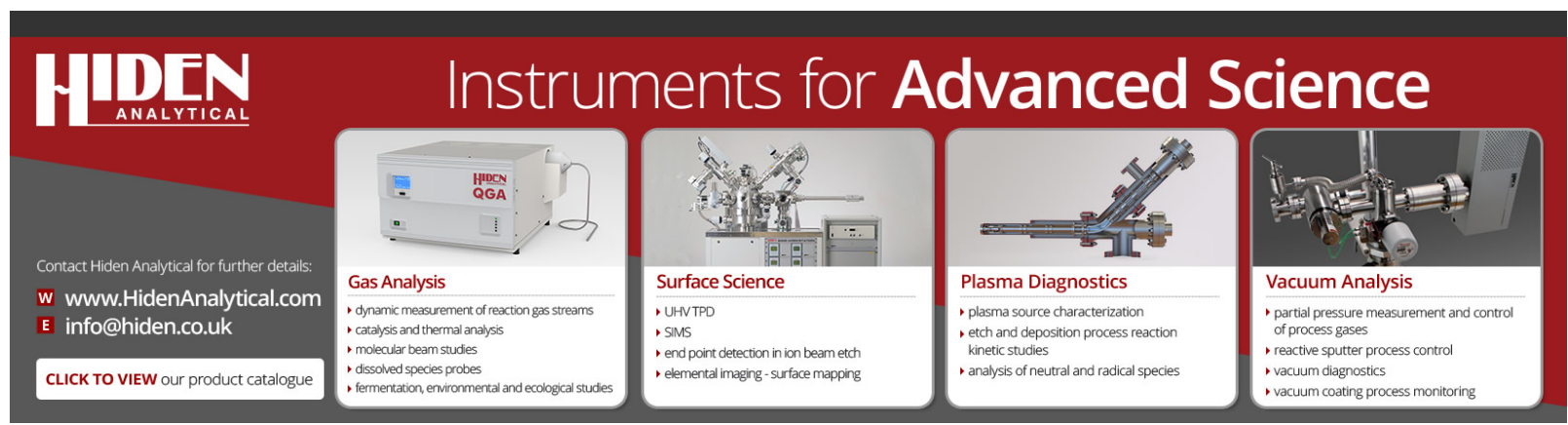




\title{
Polar anchoring energy measurement of vertically aligned liquid-crystal cells
}

\author{
Xiangyi Nie \\ Department of Electrical and Computer Engineering, University of Central Florida, \\ Orlando, Florida 32816 \\ Yi-Hsin Lin \\ College of Optics and Photonics, University of Central Florida, Orlando, Florida 32816 \\ Thomas X. Wu, Haiying Wang, and Zhibing Ge \\ Department of Electrical and Computer Engineering, University of Central Florida, \\ Orlando, Florida 32816 \\ Shin-Tson $\mathrm{Wu}^{\mathrm{a})}$ \\ College of Optics and Photonics, University of Central Florida, Orlando, Florida 32816
}

(Received 14 February 2005; accepted 4 May 2005; published online 7 July 2005)

\begin{abstract}
An electric-field method is developed for measuring the polar anchoring energy of vertically aligned nematic liquid-crystal cells. Both theoretical analyses and confirming experimental results are presented. Vertically aligned liquid-crystal cells with buffed polyimide alignment layers are used to investigate the measurement techniques. Based on the voltage-dependent transmittance of the liquid-crystal cells, a linear fitting can be obtained, leading to a precise determination of the anchoring energy. If some specific liquid-crystal material parameters are known, measuring of the cell capacitance is not necessary. () 2005 American Institute of Physics. [DOI: 10.1063/1.1942630]
\end{abstract}

\section{INTRODUCTION}

In a vertically aligned (VA) cell, the liquid-crystal (LC) molecules are aligned nearly perpendicular to the substrate surfaces. With a crossed polarizer/analyzer configuration, the VA cell exhibits an excellent contrast ratio. At normal incidence, the device's contrast ratio is insensitive to light wavelength, LC layer thickness, and operating temperature. Because of these attractive features, VA cells have been used extensively for direct-view and projection displays. The performance of a VA cell depends on the anchoring interaction between the LC monolayer and the surface alignment layers. Substrate surface treatment and the surfactant play crucial roles for achieving a uniform pretilt angle. Good molecular alignment in a VA cell is usually more difficult to obtain than that in a homogeneous cell.

The anchoring energy is an important parameter for a LC cell because it affects not only the LC alignment but also the electro-optic properties such as threshold voltage and response time. Various experimental techniques have been developed for measuring the anchoring energy. These methods can be categorized into two camps: field on and field off, depending on whether an external field is applied or not. Examples of the field-off methods are the wedge-cells technique ${ }^{1}$ and the light-scattering technique. ${ }^{2}$ In the field-on approaches, both electric-field ${ }^{3,4}$ and magnetic-field ${ }^{5}$ techniques have been considered. The field-on techniques are based on the measurements of the LC dielectric or diamagnetic Freedericksz transition effects. Usually, a strong electric field is more easily accessible and practical than a magnetic field. For this reason, the high-electric-field technique ${ }^{3}$

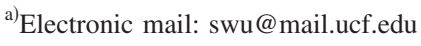

is most commonly employed for measuring the polar anchoring energy. However, some inconsistent results have been reported by different research groups that have used even the same alignment method and LC material. Afterwards, a modified electric-field technique was suggested for measuring the anchoring energy of some homogeneous cells., ${ }^{4,6}$

So far, only a few experimental methods have been developed for characterizing the anchoring energy of VA cells because of the complexity in theory and experimental technique. ${ }^{7}$ The performance of a VA-liquid-crystal display (LCD) strongly depends on the effective control of surface treatment, therefore it is essential to develop a simple and reliable technique for measuring the anchoring energy of VA LC cells.

The objective of this article is to develop a reliable electric-field technique for measuring the anchoring energy of VA cells. In Sec. II, we derive the theoretical expressions which lay down the foundation for the experimental techniques. In Sec. III, we perform confirming experiments and obtain the anchoring energy data for various VA LC cells. These results are useful for understanding the basic surface interaction mechanisms between the LC molecules and the alignment agents. Using this practical technique, we can select a proper alignment method to improve the VA-LCD performance.

\section{THEORY}

Figure 1 shows the vertical alignment of nematic LC molecules confined between two identical electrodes located at $z=0$ and $z=d$. Without electric fields, the pretilt angle $\theta_{p}$ is close to $\pi / 2$, which defines the minimum surface anchoring potential of LC directors on the substrates. Once the applied 


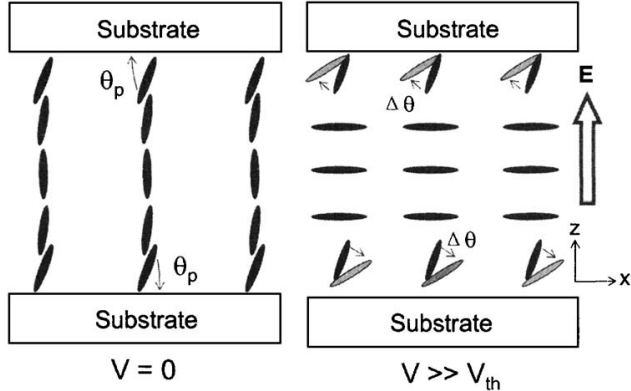

FIG. 1. Schematic drawing of the LC director distribution of a VA cell. Left: $V=0$ and right: $V \gg V_{\text {th }}$. The LC directors near the substrate surfaces could be reoriented when $V \gg V_{\text {th }}$ and the reorientation depends on the anchoring energy $W$.

voltage exceeds a threshold, the LC directors are reoriented in the $x-z$ plane. The LC molecules near the substrate surfaces are usually considered as hard anchored in theory. However, in reality, these boundary layers will also rotate and make a small contribution to the overall phase retardation if the external field is sufficiently high. In a specific voltage range, we find that the anchoring energy can be extracted through the simple phase-retardation measurement.

Our goal is to determine anchoring energy from the LC director response to the external electric field. In theory, the LC director distribution profile can be obtained by minimizing the free energy of the cell. In a voltage-on state, let us assume that the LC directors near the substrate surfaces are reoriented uniformly in the $x-z$ plane and both substrates are treated identically, the free energy per unit area of the VA LC cell can be written as

$$
\begin{aligned}
F= & \int_{0}^{d} \frac{1}{2}\left[\left(K_{11} \cos ^{2} \phi+K_{33} \sin ^{2} \phi\right)\left(\frac{d \phi}{d z}\right)^{2}-\boldsymbol{D} \cdot \boldsymbol{E}\right] d z \\
& +f_{s}(0)+f_{s}(d)
\end{aligned}
$$

The integral term in Eq. (1) represents the bulk free-energy density where $K_{11}$ and $K_{33}$ are the splay and bend elastic constants, respectively, $\phi$ is the angle between the LC director and the substrate rubbing direction ( $x$ axis), $d$ is the cell gap, and $\boldsymbol{D} \cdot \boldsymbol{E} / 2$ denotes the electric free-energy density. The last two terms $f_{s}(0)$ and $f_{s}(d)$ represent the anchoring energy density on the bottom and top boundaries. From RapiniPapoular model, ${ }^{8} f_{s}(0)$ and $f_{s}(d)$ can be represented by

$$
f_{s}=\frac{1}{2} W \sin ^{2}\left(\theta-\theta_{p}\right),
$$

provided that the electric-field-induced surface LC reorientation is not too far from the initial pretilt angle, i.e., $\Delta \theta=\theta$ $-\theta_{p}$ is small, where $\theta=\phi(0)=\phi(d)$ is the surface LC director orientation in a voltage-on state, $\theta_{p}$ is the pretilt angle, and $W$ is the anchoring strength coefficient, also called as "anchoring energy." When $\Delta \theta$ is small, e.g., $\Delta \theta<0.3 \mathrm{rad}, \sin (\Delta \theta)$ $\sim \Delta \theta$ and Eq. (2) can be simplified to $f_{s}=\frac{1}{2} W(\Delta \theta)^{2}$. The requirement of small $\Delta \theta$ imposes that $V \leqslant V_{\max }$; the maximum voltage $V_{\max }$ is found to be proportional to the product of $\Delta \theta W d .^{6}$ Since $\Delta \theta$ cannot be too large, a larger $W d$ value would allow us to obtain a higher $V_{\max }$. If a VA cell has a weak anchoring strength (i.e., small $W$ ), then we should use

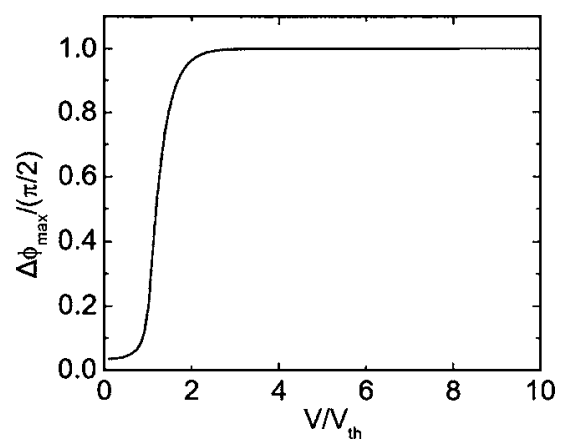

FIG. 2. Numerical simulation for the voltage-dependent $\Delta \phi_{\max }$, which validates the assumption of $\Delta \phi_{\max } \approx \pi / 2$ when $V>4 V_{\mathrm{th}}$. The LC mixture used for simulation is Merck MLC-6608: $K_{11}=16.7 \mathrm{pN}, K_{33}=18.1 \mathrm{pN}, \varepsilon_{\|}=3.6$, $\varepsilon_{\perp}=7.8, \gamma_{1}=0.186 \mathrm{Pas}, n_{o}=1.475$, and $n_{e}=1.558$.

a thick cell gap in order to improve the measurement accuracy. For display devices, their cell gap is usually thin $(d$ $<5 \mu \mathrm{m}$ ), but their surface anchoring is strong. As a result, a thin display cell can still be used for the anchoring energy measurement.

In the high-electric-field regime, the LC directors in the middle layer are fully reoriented, that is $(\Delta \phi)_{\max } \sim \pi / 2$. Under this condition, the LC cell can be treated as a semiinfinite plane during mathematical derivations. Figure 2 plots the numerical simulation of the voltage-dependent $(\Delta \phi)_{\max }$. From Fig. 2, the assumption $(\Delta \phi)_{\max } \sim \pi / 2$ is valid only when $V$ is approximately $\geqslant 4 V_{\text {th }}$. This implies that during anchoring energy experiment the applied voltage should exceed a minimum voltage $V_{\min }$ which is four times the threshold voltage. From the above analyses, the anchoring energy can be extracted only in a specific voltage range: $4 V_{\text {th }} \leqslant V$ $\leqslant V_{\max }$.

From Eq. (1), by using the variational calculus the Euler-Lagrange equation for a VA LC cell can be written as ${ }^{3}$

$$
\begin{aligned}
\frac{d}{d z}\left[\left(K_{11} \cos ^{2} \phi+K_{33} \sin ^{2} \phi\right)\left(\frac{d \phi}{d z}\right)^{2}\right. & \\
\left.-\frac{D^{2}}{\varepsilon_{0}\left(\varepsilon_{\|} \sin ^{2} \phi+\varepsilon_{\perp} \cos ^{2} \phi\right)}\right] & =0,
\end{aligned}
$$

and at $z=0$,

$\frac{d f_{s}(\theta)}{d \theta}=\left.\left(K_{11} \cos ^{2} \theta+K_{33} \sin ^{2} \theta\right) \frac{d \phi}{d z}\right|_{z=0}$,

which represents the torque balance at the LC/substrate interface.

For a normally incident beam with wavelength $\lambda$, the phase retardation $R$ of the LC cell can be calculated as follows:

$$
R=\frac{2 \pi}{\lambda} \int_{0}^{d}\left(n_{\mathrm{eff}}-n_{0}\right) d z .
$$

In Eq. (5), $n_{\mathrm{eff}}=n_{o} /\left(1-\nu \cos ^{2} \phi\right)^{1 / 2}$ and $\nu=\left(n_{e}^{2}-n_{o}^{2}\right) / n_{e}^{2}$, where $n_{o}$ and $n_{e}$ are the refractive indices of the ordinary and extraordinary rays, respectively.

Based on the above-mentioned approximations, i.e., small $\Delta \theta$ and $(\Delta \phi)_{\max } \sim \pi / 2$, we derive the following linear equation for a VA LC cell with a pretilt angle $\theta_{p} \approx \pi / 2$ : 


$$
\frac{R}{R_{0}}=-\frac{1}{C V} \frac{\xi}{\Delta n} I\left(b, \gamma, \nu, \frac{\pi}{2}\right)+\left(1+\frac{2 K_{33}}{W d}\right)
$$

where $\xi=\left(\varepsilon_{0} \varepsilon_{\perp} S / d\right) \pi \sqrt{K_{33} / \Delta \varepsilon}$, and

$$
I(b, \gamma, v, \theta)=\frac{2}{\pi} \int_{0}^{\theta} \frac{1-\nu+(1-\nu)^{1 / 2}}{1-\nu \cos ^{2} \phi+\left(1-\nu \cos ^{2} \phi\right)^{1 / 2}} \frac{\left(1+\gamma \cos ^{2} \phi\right)^{2}\left(1+b \sin ^{2} \phi\right)^{1 / 2}}{\sin \phi} d \phi .
$$

In Eq. (6), $R_{o}$ is the maximum phase retardation of the VA cell, $R / R_{o}$ is the normalized phase retardation, $C$ is the LC cell capacitance which is dependent on the applied voltage $V$, $\Delta n=n_{e}-n_{o}$ is the LC birefringence, $\Delta \varepsilon(<0)$ is the dielectric anisotropy of the negative LC material, $\varepsilon_{\perp}$ is the dielectric constant perpendicular to the LC directors, $S$ is the electrode area of the LC cell, $\gamma=\left(K_{11}-K_{33}\right) / K_{33}$, and $b=(\Delta \varepsilon) / \varepsilon_{0} \varepsilon_{\perp}$ $<0$. The term $(\xi / \Delta n) I(b, \gamma, \nu, \pi / 2)$ can be treated as a constant since all the related parameters are independent of voltage. In Eq. (6), $R / R_{o}$ is expected to be a linear function of $1 / C V$ in a specific voltage regime. Through a linear fitting in this voltage range, the anchoring energy $W$ can be determined from the intercept at $1 / C V \rightarrow 0$.

Multiplying both sides of Eq. (6) by $C V$, we derive

$$
\left(\frac{R}{R_{0}}-1\right)(C V)=\frac{2 K_{3}}{W d}(C V)-\frac{\xi}{\Delta n} I\left(b, \gamma, \nu, \frac{\pi}{2}\right) .
$$

If we plot $\left[\left(R / R_{0}\right)-1\right](C V)$ as a function of $C V, W$ can be determined from the slope of the curve, provided that $K_{33}$ and $d$ are known. As will be shown below, Eq. (7) is easier to fit than Eq. (6) because the voltage range for a linear fitting is more obvious. Please note that Eqs. (6) and (7) are valid only in the $V_{\min } \leqslant V \leqslant V_{\max }$ region, as mentioned previously.

In Eqs. (6) and (7), the LC cell capacitance $C$ is also dependent on the LC director distribution and the applied voltage $V$. It is known that near the electrode edges, fringing field-induced LC reorientation is slightly different from that in the bulk. However, in most experiments the electrode area is large $\left(>1 \mathrm{~cm}^{2}\right)$ so that the fringing field-induced LC deformation can be ignored. In the high-voltage regime, the $\mathrm{LC}$ cell capacitance is not a constant. Rather, the product of $C V$ is a linear function of $V: 6,9,10$

$$
C V=C_{\infty}\left(V-V^{\prime}\right) \text {, }
$$

where $C_{\infty}$ is the VA LC cell capacitance at $V \rightarrow \infty$. This equation predicts that the plot of $C V$ against $V$ is a straight line (with slope $C_{\infty}$ ) when $V \gg V_{\text {th }}$. For a VA cell, the $V^{\prime}$ in Eq. (8) is related to the threshold voltage as

$$
V^{\prime}=\alpha\left(1-\frac{\varepsilon_{\|}}{\varepsilon_{\perp}}\right) V_{\mathrm{th}}
$$

where $\quad \alpha=1 / \pi \int_{0}^{1} \sqrt{[(1+r)(1+k x)] / x(1+r x)} d x$, and $\quad r$ $=\left(\varepsilon_{\perp} / \varepsilon_{\|}\right)-1$.

If the LC material parameters are known, $V^{\prime}$ can be calculated easily and the undesired capacitance measurement can be avoided through adopting Eq. (8). Under such a circumstance, Eq. (7) can be simplified to

$$
\left(\frac{R}{R_{0}}-1\right)\left(V-V^{\prime}\right)=\frac{2 K_{33}}{W d}\left(V-V^{\prime}\right)-\frac{\xi}{C_{\infty} \Delta n} I\left(b, \gamma, \nu, \frac{\pi}{2}\right) .
$$

The anchoring energy is accessible through a linear fitting of the above equation.

\section{EXPERIMENT}

Figure 3 depicts the experimental setup for measuring the anchoring energy of a VA cell. The LC cell is placed between a pair of crossed polarizer and analyzer. The optical axis of the cell is oriented at $45^{\circ}$ with respect to the polarizer. The output transmittance is measured by a computercontrolled LABVIEW data acquisition system. The transmittance of such a cell can be expressed as

$$
I=I_{o} \sin ^{2}\left(\frac{R}{2}\right)
$$

where $I_{o}$ is the incident light intensity. The experiments are carried out at room temperature $\left(T \sim 22^{\circ} \mathrm{C}\right)$ and $\lambda$ $=633 \mathrm{~nm}$. From the light transmittance measurement, the LC director profile and phase retardation can be obtained and the anchoring energy extracted, as described below.

In our studies, various VA cells and negative LC materials are measured. To illustrate the measurement processes, we only show the experimental results of a 7.0- $\mu \mathrm{m}$ buffed polyimide VA cell filled with Merck MLC-6608 negative LC mixture. In the first step, we measure the light transmittance of the VA cell and obtain the voltage-dependent phase retardation. For a VA cell, the maximum phase retardation $R_{o}$ cannot be accessed accurately by simply increasing the voltage because the boundary layers are impossible to be reoriented completely even at a high voltage. Thus, the extrapolation method ${ }^{10}$ needs to be employed in order to obtain $R_{o}$.

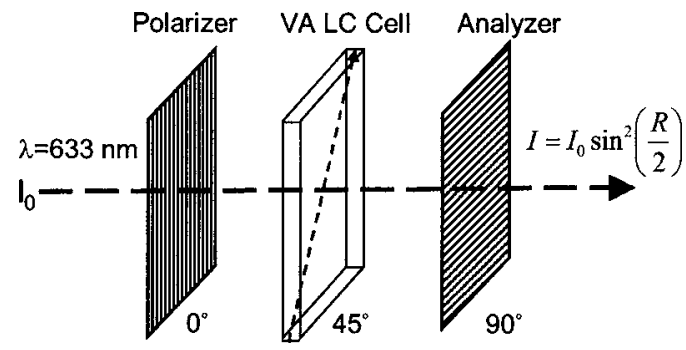

FIG. 3. Experimental setup for measuring the anchoring energy of VA cells. A VA cell is placed between crossed polarizer and analyzer. The optical axis of the VA cell is oriented at $45^{\circ}$ with respect to the polarizer. 


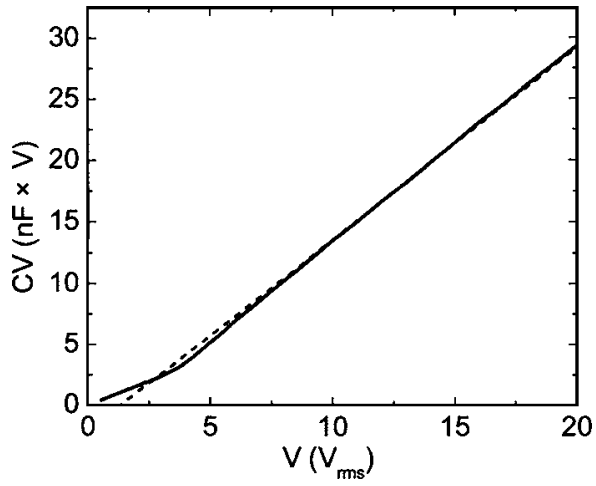

FIG. 4. Voltage-dependent $C V$ curve of a 7- $\mu \mathrm{m}$ VA cell filled with MLC6608. In the high-voltage region, a linear curve is observed and the intercept at $C V=0$ is $1.24 V_{\mathrm{rms}}$.

For the tested 7.0- $\mu \mathrm{m}$ MLC-6608 VA cell, $R_{o}$ is found to be $1.982 \pi$. In the second step, we measure the voltagedependent LC cell capacitance using a computer-controlled Displaytech APT-III instrument. Figure 4 depicts the measurement results. Indeed, the measured capacitance results confirm that $C V$ is linearly dependent on $V$ in the highvoltage region, as described by Eq. (8). Through a linear fitting in the high-voltage region, an intercept of $1.24 \mathrm{~V}$ is obtained at $C V=0$.

Figure 5 plots $R / R_{o}$ as a function of $1 / C V$, according to Eq. (6). A linear curve is observed between 8 and $20 V_{\text {rms. }}$. The projected intercept at $1 / C V=0$ is 1.017 , which leads to an anchoring strength of $W=(3.0 \pm 0.2) \times 10^{-4} \mathrm{~J} / \mathrm{m}^{2}$. This extrapolation method works reasonably well in theory and the obtained results are accurate in some cases. However, this intercept extrapolation method has two disadvantages: the intercept is usually too close to 1 and the linearity exists only in a specific voltage regime. At a high voltage, the possible nonlinear behavior of the function is masked in appearance by the reciprocal $1 / \mathrm{CV}$. These two factors limit the accuracy of this method.

To find an easier and more reliable fitting process, we develop a slope fitting method. The variable $C V$ would enable a more obvious fitting voltage range than $1 /(C V)$. Figure 6 plots the measured $R / R_{o}-1$ as a function of $C V$, according to Eq. (7). The result shows that a good linear fitting

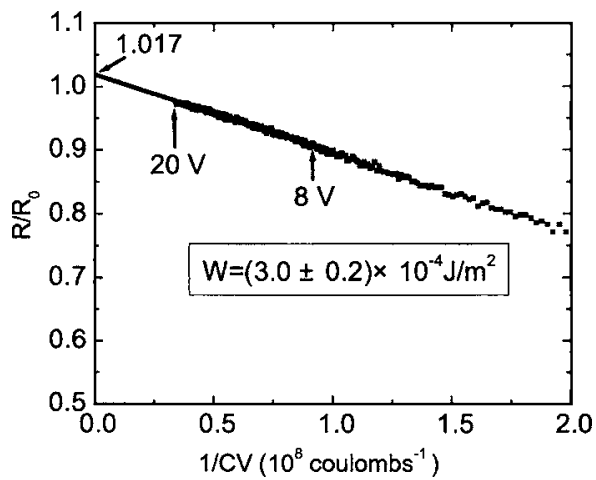

FIG. 5. Illustration of the intercept extrapolation method described in Eq. (6). A linear fitting is obtained in the $V>8 V_{\mathrm{rms}}$ region. The intercept at $1 / C V=0$ leads to $W=(3.0 \pm 0.2) \times 10^{-4} \mathrm{~J} / \mathrm{m}^{2}$. A $7-\mu \mathrm{m}$ VA cell filled with MLC-6608 is used as an example.

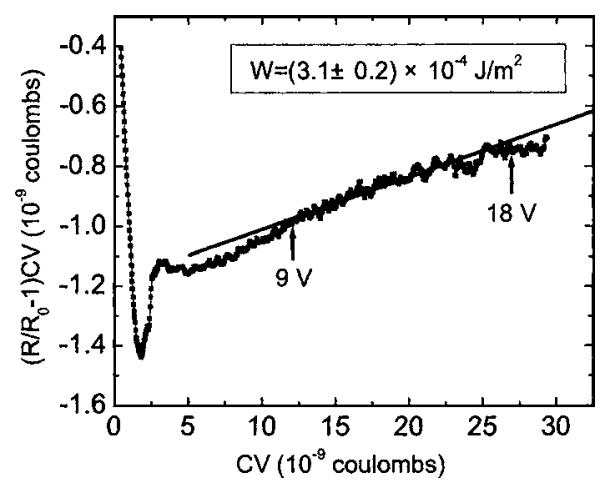

FIG. 6. Illustration of the slope fitting method according to Eq. (7). A linear fitting between $V=9 V_{\text {rms }}$ and $18 V_{\text {rms }}$ is observed, and the anchoring energy is deduced as $W=(3.1 \pm 0.2) \times 10^{-4} \mathrm{~J} / \mathrm{m}^{2}$. A $7-\mu \mathrm{m}$ VA cell filled with MLC6608 is tested as an example.

is obtained between 9 and $18 V_{\text {rms }}$. By fitting the slope of the linear curve, an anchoring strength of $W=(3.1 \pm 0.2)$ $\times 10^{-4} \mathrm{~J} / \mathrm{m}^{2}$ is deduced. The threshold voltage of MLC$6608 \mathrm{LC}$ material is $V_{\mathrm{th}} \sim 2.14 V_{\mathrm{rms}}$, which gives a minimum voltage of $V_{\min }=4 V_{\mathrm{th}} \sim 8.56 V_{\mathrm{rms}}$. From our theoretical analyses shown in Sec. II, a linear curve is predicted in the $4 V_{\text {th }}$ $\leqslant V \leqslant V_{\max }$ range. This prediction is validated as observed from Fig. 6.

For accurate determination of anchoring energy, the voltage-dependent LC cell capacitance should be taken into consideration. However, in situ capacitance measurement can be tedious and time consuming. If we could use an analytical form of the voltage-dependent capacitance instead of using the actual experimental data, Eq. (10) provides a convenient method for measuring $W$ without the variable $C$. For the 7.0- $\mu \mathrm{m}$ VA cell, $V^{\prime}$ is calculated to be $0.96 \mathrm{~V}$, which is close to the $1.24 \mathrm{~V}$ deduced from Fig. 4. Our results in Fig. 7 show that the curve is less linear than that shown in Fig. 6. The obtained anchoring strength $W=(3.2 \pm 0.3) \times 10^{-4} \mathrm{~J} / \mathrm{m}^{2}$ is $\sim 3 \%$ higher than that obtained from Fig. 6. Although this alternative method mentioned in Eq. (10) can skip the cell capacitance measurement, it is somewhat less accurate because the theoretical instead of experimental capacitance value is used.

\section{DISCUSSION}

Various techniques for measuring the polar anchoring energy have been developed and many experimental results

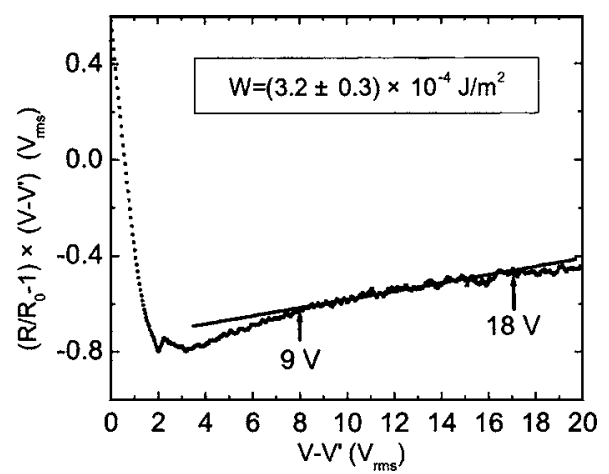

FIG. 7. Illustration of the slope fitting method according to Eq. (10). A linear fitting and a measurement result of $W=(3.2 \pm 0.3) \times 10^{-4} \mathrm{~J} / \mathrm{m}^{2}$ are obtained for the 7- $\mu \mathrm{m}$ MLC-6608 VA cell. The LC cell capacitance measurement is avoided by adopting Eq. (8). 
of different LC materials and substrates have been reported previously. However, reproducibility is a serious concern. Several research groups have derived different results even with similar or the same LC materials and substrates. This is due to the intrinsic complexity of anchoring interaction between LC materials and substrate surfaces. ${ }^{11}$

For accurate measurement of anchoring energy, the experimental setup, cells, and LC materials all require careful preparations. Light leakage from experimental setup needs to be minimized. Environment temperature may also greatly influence the results since anchoring energy increases with decreasing temperature. ${ }^{12}$ In addition, the accuracy of anchoring energy measurement depends on the LC layer thickness. The measured result of a thicker cell is usually smaller than that of a thin cell. This effect has been discussed in Ref. 13 and a possible reason is ionic effect. With electric field, ions in the LC cells are adsorbed at opposite interfaces which cause additional space-charge field. This field may influence the LC directors' reorientation. Such an ion-induced spacecharge field is difficult to calculate accurately. The overall effect is that a smaller anchoring energy is obtained for a thicker LC cell. This observation is consistent for both homogeneous and VA cells.

Thick cells are usually desired for electric-field techniques. In our proposed VA anchoring energy measurement technique, thick cells may lead to a large $V_{\max }$ and a wide voltage range between $V_{\min }$ and $V_{\max }$. If the anchoring is too weak and the cell is not thick enough, the curve in the $V$ $>V_{\min }$ region tends to be nonlinear. The reason is that $\Delta \theta$ is large and the assumption $\sin (\Delta \theta) \approx \Delta \theta$ is no longer valid. In this case, a linear fitting over $V_{\min }$ cannot be obtained. In most experiments, the polyimide (PI) alignment film is $\sim 60-80 \mathrm{~nm}$ which is much thinner than the LC cell gap (a few microns). Thus, its contribution to the experimental results is very negligible.

The in-plane inhomogeneity of LC alignment may also contribute to the error of the experimental measurements. In theory, the VA LC directors are all in $x-z$ plane, that is, $n(y)=0$. However, if a VA cell is not aligned well, then $n(y)$ may not be close to zero. In the voltage-on state, the LC directors on the substrates will not be reoriented only in the $x-z$ plane. Under this condition, the transmission of the VA
LC cell does not follow the description of Eq. (11). Serious in-plane inhomogeneous alignment of VA cells may result in a large discrepancy in measurement. The quality of VA alignment can be checked by running the transmittance between crossed and parallel polarizers. Between crossed polarizers, the VA cell should exhibit a very high contrast ratio at normal incidence. In a voltage-on state, a good VA cell should also exhibit a good dark state at a given voltage and a laser wavelength under the parallel polarizer/analyzer configuration.

\section{CONCLUSION}

We have developed an electric-field technique for measuring the polar anchoring energy of vertically aligned nematic liquid crystals. The anchoring energy can be determined by measuring the voltage-dependent phase retardation of a VA liquid-crystal cell. This method is simple and reliable. Widespread application of this measurement technique in the LCD industry is foreseeable.

\section{ACKNOWLEDGMENTS}

The authors would like to acknowledge C. H. Wen and Dr. S. Gauza for their technical assistance and useful discussions. This work is supported by Toppoly Optoelectronics (Taiwan).

${ }^{1}$ G. Barbero, N. V. Madhusudana, and G. Durand, J. Phys. (France) Lett. 45, L-613 (1984).

${ }^{2}$ T. Marusiy, Yu. Reznikov, and V. Reshetnyak, Sov. Phys. JETP 64, 502 (1986).

${ }^{3}$ H. Yokoyama and H. A. van Sprang, J. Appl. Phys. 57, 4520 (1985).

${ }^{4}$ Yu. A. Nastishin, R. D. Polak, S. V. Shiyanovskii, and O. D. Lavrentovich, Appl. Phys. Lett. 75, 202 (1999).

${ }^{5}$ K. H. Yang and C. Rosenblatt, Appl. Phys. Lett. 43, 62 (1983).

${ }^{6}$ Yu. A. Nastishin, R. D. Polak, S. V. Shiyanovskii, V. H. Bodnar, and O. D. Lavrentovich, J. Appl. Phys. 86, 4199 (1999).

${ }^{7}$ F. Yang, L. Ruan, and J. R. Sambles, J. Appl. Phys. 88, 6175 (2000).

${ }^{8}$ A. Rapini and M. Papoular, J. Phys. (Paris), Colloq. 30, C-4 (1969).

${ }^{9}$ K. R. Welford and J. R. Sambles, Mol. Cryst. Liq. Cryst. 147, 25 (1987).

${ }^{10}$ I. C. Khoo and S. T. Wu, Optics and Nonlinear Optics of Liquid Crystals (World Scientific, Singapore, 1993).

${ }^{11}$ G. Carbone and C. Rosenblatt, Phys. Rev. Lett. 94, 057802 (2005).

${ }^{12}$ C. Rosenblatt, J. Phys. (Paris) 45, 1087 (1984).

${ }^{13}$ L. M. Blinov and V. G. Chigrinov, Electrooptic Effects in Liquid Crystal Materials (Springer, New York, 1994). 